Pacific Journal of Mathematics

UNIQUENESS IN A DOUBLY CHARACTERISTIC CAUCHY

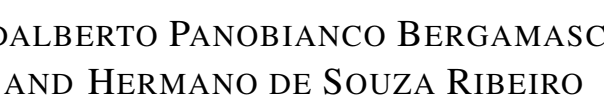




\title{
UNIQUENESS IN A DOUBLY CHARACTERISTIC CAUCHY PROBLEM
}

\author{
Adalberto P. Bergamasco and Hermano de Souza Ribeiro
}

This article studies uniqueness, in the class of distributions, of solutions of the Cauchy problem for a class of degenerate hyperbolic second order equations, when the initial curve contains a doubly characteristic point. The techniques employed are Carleman estimates and the concatenation method.

1. Introduction. This work is concerned with the uniqueness in the characteristic Cauchy problem for operators with double characteristics at a point of the initial curve.

We obtain an extension of results in [T2] and [BP]: these authors study uniqueness across $y=0$ for the operator

$$
\left(D_{x}-x D_{y}\right)\left(D_{x}+x D_{y}\right)-c D_{y}
$$

throughout the article we use the notation $D_{x}=\partial / \partial x, D_{y}=\partial / \partial y$.

Our work has an overlapping with $[\mathbf{N}]$ and $[\mathbf{K}]$. In $[\mathbf{N}]$, the study is made in the context of hyperfunction theory and results are proven for operators, e.g., of the type: $\left(D_{x}-x^{k} D_{y}\right)\left(D_{x}+x^{k} D_{y}\right)-c x^{k-1} D_{y}$, $k$ a natural number; our method of proof uses only the theory of distributions. In [K], operators, e.g., like

$$
P(a, b)=\left[D_{x}-a(x) D_{y}\right]\left[D_{x}+a(x) D_{y}\right]+b(x) D_{y},
$$

where $a$ has a zero of order one at zero, are dealt with; in our work, if, say, $b$ is non-negative then $a$ is allowed to vanish to an arbitrary odd order $k$ at zero.

Section 2 contains the proof of the Carleman estimates which yield the uniqueness across $y=0$, in the class $C^{2}$, for the operator $P(a, b)$, under suitable assumptions. The results of this section are more general than what we needed in our applications. In the beginning of $\S 3$, we specialize our operator $P(a, b)$ to the case $a(x)=-a x^{k}$, $b(x)=-c x^{k-1}, k$ odd, and, by using the concatenations in [GT], we prove uniqueness, in the class $C^{m}$, where $m$ depends on $c$ and $c$ avoids a certain sequence of real numbers. When $c$ takes on such values, it is possible to prove that there is non-uniqueness, even in the class $C^{\infty}$ 
(see [N]). The result in Theorem 3.2 refers to uniqueness when the initial curve lies midway between the pair of characteristics through the origin.

Section 3 also contains a result of uniqueness-not covered by [N]-for operators of the type

$$
\left(D_{x}+a x^{k} D_{y}\right)\left(D_{x}+b x^{l} D_{y}\right)-\left(c x^{k-1}+d x^{l-1}\right) D_{y}
$$

where $k, l$ are odd natural numbers and $a, b, c, d$ are real numbers satisfying certain conditions (see Theorem 3.3).

In $\S 4$, we extend the method used in [BP] and we show how to obtain uniqueness in the class of distributions from uniqueness in the class $C^{m}$ for some $m$, in the case of a certain type of operators among which are all the above mentioned operators. It is interesting to compare Theorem 4.1 with Theorem 4.4 .8 in [H].

The authors hope to prove, in a forthcoming publication, the uniqueness in the Cauchy problem for operators such as

$$
\left[D_{x}+a(x, y) D_{y}\right]\left[D_{x}+b(x, y) D_{y}\right]-c(x, y) D_{x}-d(x, y) D_{y}-e(x, y)
$$

where both $a(x, 0)$ and $b(x, 0)$ have zeros of orders greater than one at $x=0$. This will be accomplished by means of certain approximate concatenations since we haven't been able to find exact ones in this more general set up.

2. Carleman estimates and uniqueness in the class $C^{2}$ for the operator $P(a, b)=\left(D_{x}-a(x) D_{y}\right)\left(D_{x}+a(x) D_{y}\right)+b(x) D_{y} ; a \in C^{1}(\mathbf{R}, \mathbf{R}), b \in$ $C^{0}(\mathbf{R}, \mathbf{R})$.

Proposition 2.1. Assume that the following condition is satisfied:

(H) There exist $M>0$ and $r>0$ such that

$$
g(x)=M[a(x)]^{2}+a^{\prime}(x)+b(x) \geq 0, \quad x \in[-r, r]
$$

where $a^{\prime}(x)$ is the derivative of $a(x)$.

Then the following estimates hold for all $v \in C_{c}^{2}(X, \mathbf{C})$, where $X \subset$ $\left\{(x, y) \in \mathbf{R}^{2}:|x| \leq r\right\}$ is a nonempty bounded open subset of $\mathbf{R}^{2}$ :

(T1) If $P^{\#}(a, b, t)=\exp (t y) P(a, b) \exp (-t y)$, then for $t \geq M$

$$
\operatorname{Re}\left\langle P^{\#}(a, b, t) v, D_{y} v\right\rangle \geq C \iint_{X} g|v|^{2} d x d y
$$

where $\langle$,$\rangle is the usual inner product of L^{2}(X, \mathbf{C})$ and where the constant $C>0$ is independent of $v$. 
(T2) For the same constant $C>0$ and for $t \geq M$,

$$
\begin{aligned}
& C \iint_{X} g(x) \exp (2 t y)|v|^{2} d x d y \\
& \quad \leq \iint_{X} \exp (2 t y)\left[\left|P(a, b) v \cdot \overline{D_{y} v}\right|+t|P(a, b) v \cdot \bar{v}|\right] d x d y .
\end{aligned}
$$

Proof. First we use (T1) to prove (T2). We have

$$
\begin{array}{rl}
C \iint_{X} & g(x) \exp (2 t y)|v|^{2} d x d y \\
\quad \leq & \operatorname{Re}\left\langle P^{\#}(a, b, t)(v \exp (t y)), D_{y}(v \exp (t y))\right\rangle \\
\quad & \operatorname{Re}\left\langle\exp (t y) P(a, b)(v), \exp (t y)\left(t v+D_{y} v\right)\right\rangle \\
\quad \leq\left|\iint_{X} \exp (2 t y)\left[P(a, b)(v) \cdot \overline{D_{y} v}+t P(a, b)(v) \cdot \bar{v}\right] d x d y\right| \\
\quad \leq \iint_{X} \exp (2 t y)\left[\left|P(a, b)(v) \cdot \overline{D_{y} v}\right|+t|P(a, b)(v) \cdot \bar{v}|\right] d x d y .
\end{array}
$$

We shall now prove (T1). We first get

$$
\int_{-\infty}^{+\infty}|v|^{2} d y \leq C^{-1} \int_{-\infty}^{+\infty}\left|D_{y} v\right|^{2} d y
$$

where the constant $C>0$ is independent of $v \in C_{c}^{2}(X, \mathbf{C})$.

Then

$$
C g(x) \int_{-\infty}^{+\infty}|v|^{2} d y \leq g(x) \int_{-\infty}^{+\infty}\left|D_{y} v\right|^{2} d y
$$

and

$$
\begin{gathered}
C \iint_{X} g|v|^{2} d x d y \leq \iint_{X} g\left|D_{y} v\right|^{2} d x d y \\
\quad=\iint_{X}\left[M a^{2}+a^{\prime}+b\right]\left|D_{y} v\right|^{2} d x d y \\
\quad \leq \iint_{X}\left[2 t a^{2}+a^{\prime}+b\right]\left|D_{y} v\right|^{2} d x d y \\
=\operatorname{Re}\left\langle P^{\#}(a, b, t)(v), D_{y} v\right\rangle
\end{gathered}
$$

since $t \geq M$. 
THEOREM 2.1. Assume that the following conditions are satisfied:

(H1) There exist $M>0$ and $r>0$ such that

$$
g(x)=M[a(x)]^{2}+a^{\prime}(x)+b(x) \geq 0, \quad x \in[-r, r]
$$

and $g$ is not identically zero on any subinterval of $[-r, r]$.

(H2) $X \subset\left\{(x, y) \in \mathbf{R}^{2}:|x| \leq r\right\}$ is a nonempty bounded open subset of $\mathbf{R}^{2}$ and $F$ is a relatively closed subset of $X$ whose intersection with $X_{+}=\{(x, y) \in X: y \geq 0\}$ is a compact set $K$.

Then the following holds

(T) There exists an open neighborhood $U$ of $K$ such that every function $u \in C^{2}(X, \mathbf{C})$ satisfying

$$
\text { (1) } P(a, b) u=0 \quad \text { in } X ; \quad \text { (2) } \operatorname{supp} u \subset F
$$

also satisfies $u=0$ in $U$.

Proof. The proof uses the estimates proved in the previous section following the lines of Theorem 2.3 of [T1].

3. Concatenations and uniqueness in the class $C^{m}$ for the operator $P(a, b, c, k)=\left(D_{x}+a x^{k} D_{y}\right)\left(D_{x}+b x^{k} D_{y}\right)-c x^{k-1} D_{y}$. We are going to use

Lemma 3.1. Assume that

(H1) For $a, \mu \in \mathbf{C}$ and $k \in N$, let

$$
\begin{aligned}
Q(a, k, \mu) & =x\left(D_{x}+a x^{k} D_{y}\right)+\mu, \\
R(a, k) & =D_{x}+a x^{k} D_{y} .
\end{aligned}
$$

Then the following holds for $a, b, c \in \mathbf{C}$ and $(a-b) b \neq 0$.

(T1) $Q(a, k, \mu) P(a, b, c, k)=P\left(a, b, c^{\prime}, k\right) Q(a, k, \mu-2)$ if $c^{\prime}=c+$ $(k+1)(a-b)$ and $\mu-2=k+c /(a-b)$.

(T2) $x P(a,-a, c, k)-R(-a, k) Q(a, k, \mu-2)=\left(-c^{\prime} / 2 a\right) R(a, k)$, if $a \neq 0$.

Proof. For (T1) see [GT]. A simple computation shows that (T2) also holds.

LEMMA 3.2. For $m \geq 2$ and $c \in \mathbf{C}$, consider the statement:

$S(m, c)$ : every function $u \in C^{m}(X, \mathbf{C})$ satisfying

(1) $P(a, b, c, k) u=0$ in $X$ with $a=-b$,

(2) supp $u \subset F$

vanishes identically in $U$ (here, $X, F$ and $U$ are as in Theorem 2.1). 
If $c^{\prime}=c+(k+1)(a-b) \neq 0$ and $\mu-2=k+c /(a-b) \neq 0$ then $S\left(m, c^{\prime}\right)$ implies $S(m+1, c)$.

Proof. Let $u \in C^{m+1}(X, \mathbf{C})$ be such that

(1) $P(a, b, c, k) u=0$ in $U$ with $a=-b$,

(2) $\operatorname{supp} u \subset F$.

From $Q(a, k, \mu) P(a, b, c, k) u=P\left(a, b, c^{\prime}, k\right) Q(a, k, \mu-2) u$ it follows that

$$
P\left(a, b, c^{\prime}, k\right) Q(a, k, \mu-2) u=0 \text { in } U
$$

and therefore by hypothesis

$$
Q(a, k, \mu-2) u=0 \text { in } U .
$$

Thus, if $a \neq 0$,

$$
\begin{aligned}
0 & =x P(a,-a, c, k) u-R(-a, k) Q(a, k, \mu-2) u \\
& =\frac{-c^{\prime}}{2 a} R(a, k) u \text { in } U .
\end{aligned}
$$

Since $c^{\prime} \neq 0$,

$$
0=Q(a, k, \mu-2) u=x R(a, k) u+(\mu-2) u \text { in } U
$$

and so $(\mu-2) u=0$ in $U$; since $\mu-2 \neq 0$, we get $u=0$ in $U$. The lemma is proved.

THEOREM 3.1. Assume that the following condition is satisfied:

(H) $X$ is a nonempty bounded open subset of $\mathbf{R}^{2}$ and $F$ is a relatively closed subset of $X$ whose intersection with $X_{+}=\{(x, y) \in X: y \geq 0\}$ is a compact set $K$.

Then the following holds

(T) There exist $m \geq 2$, depending on $c$, and an open neighborhood $U$ of $K$ such that every function $u \in C^{m}(X, \mathbf{C})$ satisfying.
(1) $P(a,-a, c, k) u=0$ in $X$;
(2) $\operatorname{supp} u \subset F$

also satisfies $u=0$ in $U$, when one of the following holds:

(i) $0 \neq a, c \in \mathbf{R} ; k a+c=0$

(ii) $a, c \in \mathbf{R} ; k$ odd and $k a+c<0$

(iii) $0>a, c \in \mathbf{R} ; k$ odd and for all $j=0,1,2, \ldots c \neq-2 a j(k+1)$, $c \neq-2 a[k+j(k+1)]$.

Proof. The first two cases are consequences of Theorem 2.1; in these cases, $m=2$. In the third case, if $c$ is non-positive, we are in 
the preceding case and $m=2$. If $c>0$, let $j_{0}$ be the smallest natural number such that $c_{1}=c+2 a(k+1) j_{0}<0$. As in the preceding case, $S\left(2, c_{1}\right)$ holds. We apply Lemma $3.2 j_{0}$ times and conclude that $S\left(2+j_{0}, c\right)$ holds; in this case, $m=2+j_{0}$. The proof is complete.

THEOREM 3.2. Assume that the following condition is satisfied:

(H) $X$ is a nonempty bounded open subset of $\mathbf{R}^{2}$ and $F$ is a relatively closed subset of $X$ whose intersection with

$$
X_{a, b, k}=\left\{(x, y) \in X: y \geq\left(\frac{a+b}{2}\right) \frac{x^{k+1}}{k+1}\right\}
$$

is a compact set $K$.

Then the following holds:

(T) There exist $m \geq 2$ depending on $c$, and an open neighborhood $U$ of $K$ such that every function $u \in C^{m}(X, \mathbf{C})$ satisfying
(1)
$P(a, b, c, k) u=0 \quad$ in $X$
(2) $\operatorname{supp} u \subset F$

also satisfies $u=0$ in $U$, when one of the following holds:

(i) $a, b, c \in \mathbf{R} ; a \neq b ; k((a-b) / 2)+c=0$

(ii) $a, b, c \in \mathbf{R} ; k$ odd and $k((a-b) / 2)+c<0$

(iii) $a, b, c \in \mathbf{R} ; a-b<0 ; k$ odd and for all $j=0,1,2, \ldots c \neq$ $j(b-a)(k+1), c \neq(b-a)[k+j(k+1)]$.

TheOREM 3.3. Let $P=\left(D_{x}+p(x) D_{y}\right)\left(D_{x}+q(x) D_{y}\right)-s(x) D_{y}$, where $p, q$ and $s$ are smooth functions vanishing at $x=0$. Assume that the following condition is satisfied:

(H) There exist $M>0$ and $r>0$ such that

$$
g(x)=M\left(\frac{p(x)-q(x)}{2}\right)^{2}-\frac{p^{\prime}(x)-q^{\prime}(x)}{2}-s(x) \geq 0, \quad x \in[-r, r] .
$$

Then the following holds:

(T) If $X \subset\left\{(x, y) \in \mathbf{R}^{2}:|x| \leq r\right\}$ is a nonempty bounded open subset of $\mathbf{R}^{2}$ and $F$ is a relatively closed subset of $X$ whose intersection with

$$
\begin{aligned}
X_{p, q}=\left\{(x, y) \in X: 2 y \geq p_{1}(x)+q_{1}(x)\right. \\
\left.\quad p_{1}^{\prime}(x)=p(x), p_{1}(0)=0, q_{1}^{\prime}(x)=q_{(}(x), q_{1}(0)=0\right\}
\end{aligned}
$$

is a compact set $K$ then: "there exists an open neighborhood $U$ of $K$ 
such that every function $u \in C^{2}(X ; \mathbf{C})$ satisfying.
(1) $P u=0$ in $X$;
(2) $\operatorname{supp} u \subset F$

also satisfies $u=0$ in $U$ ".

In particular, if $p(x)=a x^{k}, q(x)=b x^{l}, s(x)=c x^{k-1}+d x^{l-1}$, where $k$ and $l$ are odd with $k<l$, then the conditions $a \neq 0, k a+2 c<0$ imply uniqueness in the class $C^{2}$ across

$$
y=\frac{a}{2} \frac{x^{k+1}}{k+1}+\frac{b}{2} \frac{x^{l+1}}{l+1} .
$$

Theorems 3.2 and 3.3 follow, after a change of variables, from Theorem 3.1.

4. Uniqueness in the class of distributions for the operator $P=$ $D_{x x}^{2}-A(x) D_{x y}^{2}-B(x) D_{y y}^{2}-C(x) D_{x}-D(x) D_{y}-E(x)$ where $A, B, C, D$, $E$ are smooth functions of the variable $x$. In this section we have three results: the first one, Theorem 4.1, shows how to obtain uniqueness in the class of distributions from uniqueness in the class $C^{m}$, for some $m \geq 2$. The other two (given without proof) are applications of it to the operator $P(a, b, c, k)$ of the preceding section.

THEOREM 4.1. Assume that the following conditions are satisfied:

(H1) $X$ is a nonempty bounded open subset of $R^{2}$ and $F$ is a relatively closed subset of $X$ whose intersection with $X_{+}=\{(x, y) \in X: y \geq 0\}$ is a compact set $K$.

(H2) There exist a natural number $m \geq 2$ and an open neighborhood $V$ of $K$ such that every $v \in C^{m}(X, \mathbf{C})$ satisfying:

(1) $P v=0$ in $X$,

(2) $\operatorname{supp} v \subset F$

vanishes identically on $V$.

Then the following holds

(T) There exists an open neighborhood $U$ of $K$ such that every distribution $u \in D^{\prime}(U)$ satisfying.

(1) $P u=0$ in $X$,

(2) $\operatorname{supp} u \subset F$

vanishes identically on $U$.

Proof. We shall follow closely the article [BP].

Preliminaries. Let $u \in D^{\prime}(X)$ be such that

(1) $P u=0$ in $X$,

(2) $\operatorname{supp} u \subset F$. 
Pick $r>0$ such that

$$
K_{3 r}=\left\{(x, y) \in R^{2}: d((x, y), K) \leq 3 r\right\} \subset X .
$$

The closure of the set

$$
\left(K_{3 r} \backslash K_{r}\right) \cap\left\{(x, y) \in R^{2}: y=0\right\}
$$

is compact and disjoint from $F$, and therefore its distance to $F$ is strictly positive.

Now choose $p<0$ with the following properties:

(i) $0<|p|<r$,

(ii) $K_{r} \cap F \cap X_{p}=K_{3 r} \cap F \cap X_{p}$, where

$$
X_{p}=\{(x, y) \in X: y>p\} .
$$

By using a partition of unity, it is possible to express $u \in D^{\prime}(X)$ as $u=u_{1}+u_{2}$ with supp $u_{1} \subset K_{2 r} \cap F$ and supp $u_{2} \subset K_{r}^{c} \cap F$. (It suffices to take $g \in C_{c}^{\infty}(X, \mathbf{R})$ with $g \equiv 0$ in $K_{2 r}^{c}$ and $g \equiv 1$ in an open neighborhood of $K_{r}$ and then take $u_{1}=g u$ and $u_{2}=(1-g) u$.) We have

(iii) (supp $\left.u_{2}\right) \cap K_{3 r} \cap X_{p}=\varnothing$.

(Indeed, by using (ii), we get

$$
\begin{aligned}
\phi & =K_{r} \cap X_{p} \cap \operatorname{supp} u_{2}=K_{r} \cap X_{p} \cap F \cap \operatorname{supp} u_{2} \\
& =K_{3 r} \cap X_{p} \cap F \cap \operatorname{supp} u_{2}=K_{3 r} \cap X_{p} \cap \operatorname{supp} u_{2} \text { ) }
\end{aligned}
$$

Notice $P u=0$ in $X$ implies $P u_{1}=-P u_{2}$ in $X$ and supp $P u_{1} \subset \operatorname{supp} u_{1} \cap$ supp $u_{2}$. Also, by (iii)

(iv) $X_{p} \cap \operatorname{supp} P u_{1}=\varnothing$.

Extend $u_{1}$ to a distribution on $R^{2}$ by setting it equal to zero outside $K_{3 r}$. By (iv), $P u_{1}=0$ in $X_{p}$. By L. Schwartz's theorem on the structure of distributions with compact support, there exist a non-negative integer $n$ and continuous functions $f_{j k}$ such that supp $f_{j k} \subset K_{3 r}$ and

(v) $u_{1}=\sum_{j+k \leq n} D_{x}^{j} D_{y}^{k} f_{j k}$

in the sense of distributions.

In order to simplify our notation we pick $b \in R$ such that

$$
K_{4 r} \subset Q=\left\{(x, y) \in R^{2}: x>b, y>b\right\} .
$$

For each continuous function $f: R^{2} \rightarrow C$ with supp $f \subset Q$, we define

$$
\begin{aligned}
& {\left[D_{x}^{-1} f\right](x, y)=\int_{b}^{x} f(t, y) d t,} \\
& {\left[D_{x}^{-1} f\right](x, y)=\int_{b}^{y} f(x, t) d t .}
\end{aligned}
$$


It is clear that $\left[D_{x}^{-1} f\right](x, y)=0$ if $x \leq b$ and that $\left[D_{y}^{-1} f\right](x, y)=0$ if $y \leq b$. It is also obvious that $D_{x}, D_{y}, D_{x}^{-1}$ and $D_{y}^{-1}$ "commute" (e.g., if $f$ is $C^{1}$, we have $\left.D_{x} D_{x}^{-1} f=D_{x}^{-1} D_{x} f=f=D_{y} D_{y}^{-1} f=D_{y}^{-1} D_{y} f\right)$. We define, for $n=1,2, \ldots, D_{x}^{-n}=\left[D_{x}^{-1}\right]^{n}$ and $D_{y}^{-n}=\left[D_{y}^{-1}\right]^{n}$. Also, $D_{x}^{0} f=D_{y}^{0} f=f$. With these notations, we may write

(v) $u_{1}=D_{x}^{n} D_{y}^{n} f$

where $f=\sum_{j+k \leq n} D_{x}^{j-n} D_{y}^{k-n} f_{j k}$. We have

$$
\operatorname{supp} f \subset\left\{(x, y) \in R^{2}: x>b+r, y>b+r\right\} \text {. }
$$

Regularization of $u_{1}$ in the variable $y$. Let $\psi \in C_{c}^{\infty}(R, R)$ with $\int_{-\infty}^{+\infty} \psi(t) d t=1$ and $\psi(t)=0$ for $|t|>1 / 2$. For each $\varepsilon>0$, we set $\psi_{\varepsilon}(t)=\varepsilon^{-1} \psi\left(\varepsilon^{-1} t\right), t \in R$ and $v_{\varepsilon}=u_{1} *^{\prime} \psi_{\varepsilon}$ where $*^{\prime}$ denotes convolution in variable $y$ only.

The fact that the coefficients of $P$ are independent of $y$ implies $P v_{\varepsilon}=P u_{1} *^{\prime} \psi_{\varepsilon}$ for $\varepsilon>0$. If $0<\varepsilon<|p|<r$ then supp $v_{\varepsilon} \subset K_{4 r} \subset Q$. Also

$$
v_{\varepsilon}=u_{1} *^{\prime} \psi_{\varepsilon}=\left(D_{x}^{n} D_{y}^{n} f\right) *^{\prime} \psi_{\varepsilon}=D_{x}^{n}\left[f *^{\prime} D_{y}^{n} \psi_{\varepsilon}\right]=D_{x}^{n} g
$$

where the function $g=f *^{\prime} D_{y}^{n} \psi_{\varepsilon}$ is continuous, has partial derivatives of any order in the variable $y$ and satisfies supp $g \subset Q$.

Substitution of $v_{\varepsilon}=D_{x}^{n} g$ in $P v_{\varepsilon}=0$ on $X_{p+\varepsilon / 2}$.

$$
\begin{aligned}
D_{x x}^{2}\left[D_{x}^{n} g\right]= & A D_{x y}^{2}\left[D_{x}^{n} g\right]+B D_{y y}^{2}\left[D_{x}^{n} g\right] \\
& +C D_{x}\left[D_{x}^{n} g\right]+D D_{y}\left[D_{x}^{n} g\right]+E D_{x}^{n} g .
\end{aligned}
$$

First Case. $n=1,2,3, \ldots$

$$
\begin{aligned}
A D_{x y}^{2}\left[D_{x}^{n} g\right]=A D_{y}\left[D_{x}^{n+1} g\right] & =D_{x}^{n+1}\left[A D_{y} g\right]-\left(\begin{array}{c}
n+1 \\
1
\end{array}\right) A^{\prime}(x) D_{x}^{n}\left[D_{y} g\right] \\
& -\sum_{j=2}^{n+1}\left(\begin{array}{c}
n+1 \\
j
\end{array}\right) D_{x}^{j} A D_{x}^{n+1-j}\left[D_{y} g\right] \\
= & D_{x}^{n+1}\left[A D_{y} g\right]-\left(\begin{array}{c}
n+1 \\
1
\end{array}\right)\left[D_{x}^{n}\left[A^{\prime} D_{y} g\right]-\sum_{j=1}^{n}\left(\begin{array}{c}
n \\
j
\end{array}\right) D_{x}^{j} A^{\prime} D_{x}^{n-j}\left[D_{y} g\right]\right] \\
& -\sum_{j=2}^{n+1}\left(\begin{array}{c}
n+1 \\
j
\end{array}\right) D_{x}^{j} A D_{x}^{n+1-j}\left[D_{y} g\right] \\
= & D_{x}^{n+1}\left[A D_{y} g+A_{1} D_{x}^{-1}\left[A^{\prime} D_{y} g\right]+\cdots+A_{n+1} D_{x}^{-n-1}\left[A^{(n+1)} D_{y} g\right]\right],
\end{aligned}
$$




$$
\begin{gathered}
B D_{y}^{2}\left[D_{x}^{n} g\right] D_{x}^{n}\left[B D_{y}^{2} g\right]-\sum_{j=1}^{n}\left(\begin{array}{c}
n \\
j
\end{array}\right) D_{x}^{j} B D_{x}^{n-j}\left[D_{y}^{2} g\right] \\
=D_{x}^{n+1}\left[D_{x}^{-1}\left[B D_{y}^{2} g\right]+B_{1} D_{x}^{-2}\left[B^{\prime} D_{y}^{2} g\right]+\cdots+B_{n} D_{x}^{-n-1}\left[B^{(n)} D_{y}^{2} g\right]\right] \\
C D_{x}^{n+1}[g]=D_{x}^{n+1}[C g]-\sum_{j=1}^{n+1}\left(\begin{array}{c}
n+1 \\
j
\end{array}\right) D_{x}^{j} C D_{x}^{n+1-j} g \\
=D_{x}^{n+1}\left[C g+C_{1} D_{x}^{-1}\left[C^{\prime} g\right]+\cdots+C_{n+1} D_{x}^{-n-1}\left[C^{(n+1)} g\right]\right] \\
D D_{y}\left[D_{x}^{n} g\right]=D_{x}^{n}\left[D D_{y} g\right]-\sum_{j=1}^{n}\left(\begin{array}{c}
n \\
j
\end{array}\right) D_{x}^{j} D D_{x}^{n-j}\left[D_{y} g\right] \\
=D_{x}^{n+1}\left[D_{x}^{-1}\left[D D_{y} g\right]+D_{1} D_{x}^{-2}\left[D^{\prime} D_{y} g\right]+\cdots+D_{n} D_{x}^{-n-1}\left[D^{(n)} D_{y} g\right]\right] \\
E D_{x}^{n}[g]=D_{x}^{n}[E g]-\sum_{j=1}^{n}\left(\begin{array}{c}
n \\
j
\end{array}\right) D_{x}^{j} E D_{x}^{n-j} g \\
=D_{x}^{n+1}\left[D_{x}^{-1}[E g]+E_{1} D_{x}^{-2}\left[E^{\prime} g\right]+\cdots+E_{n} D_{x}^{-n}\left[E^{(n)} g\right]\right] .
\end{gathered}
$$

We get $D_{x}^{n+2} g=D_{x}^{n+1} h$ or $D_{x} g=h$. Therefore, $v_{\varepsilon}=D_{x}^{n-1} h$ where $h$ has the same properties of $g$.

Second case. $n=0$.

$$
\begin{aligned}
D_{x}^{2} g= & A D_{x}\left[D_{y} g\right]+B D_{y}^{2} g+C D_{x} g+D D_{y} g+E g \\
= & D_{x}\left[A D_{y} g-D_{x}^{-1}\left[A^{\prime} D_{y} g\right]+D_{x}^{-1}\left[B D_{y}^{2} g\right]\right. \\
& \left.\quad+C g-D_{x}^{-1}\left[C^{\prime} g\right]+D_{x}^{-1}\left[D D_{y} g\right]+D_{x}^{-1}[E g]\right] .
\end{aligned}
$$

We get $D_{x}^{2} g=D_{x} h$ or $D_{x} g=h$. Therefore, $v_{\varepsilon}=D_{x}^{-1} h$ where $h$ has the same properties of $g$.

Third case. $-n=+1,+2, \ldots$.

We shall use the following "Leibniz Formula":

$$
\begin{aligned}
D_{x}^{-n}[F G] & =F D_{x}^{-n}[G]-\sum_{j=1}^{n} D_{x}^{-j}\left[D_{x}[F] D_{x}^{-n+j-1}[G]\right], \\
A D_{x y}^{2}\left[D_{x}^{-n} g\right] & =A D_{x}^{-n+1}\left[D_{y} g\right] \\
& =\sum_{j=1}^{n-1} D_{x}^{-j}\left[A^{\prime} D_{x}^{-n+j}\left[D_{y} g\right]\right]+D_{x}^{-n+1}\left[A D_{y} g\right]
\end{aligned}
$$


By applying Leibniz formula to $A^{\prime} D_{x}^{-n+1}\left[D_{y} g\right], A^{\prime} D_{x}^{-n+2}\left[D_{y} g\right], \ldots$, $A^{\prime} D_{x}^{-1}\left[D_{y} g\right]$ and to the other terms of the equation $P v_{\varepsilon}=0$ we arrive at $D_{x}^{-n+2} g=D_{x}^{-n+1} h$ or $D_{x} g=h$. Therefore, $v_{\varepsilon}=D_{x}^{-n-1} h$ where $h$ has the same properties as $g$.

Epilogue. The analysis above furnishes the inductive step in proving that for each integer $n$ there exists $g_{n}$, continuous, with supp $g_{n} \subset Q$ and

$$
v_{\varepsilon}=D_{x}^{n} g_{n} \quad \text { in } X_{p+\varepsilon / 2}
$$

$g$ being smooth in $y$.

We reach the conclusion that $v_{\varepsilon}$ restricted to $X$ is in $C^{\infty}\left(X_{p+\varepsilon / 2}, C\right)$.

Now assumption (H2) implies that $v_{\varepsilon}=0$ in $X_{p+\varepsilon / 2}$. Therefore $u_{1}=0$ in $X_{p}$ since $v_{\varepsilon}$ converges to $u_{1}$ in $X_{p}$ when $\varepsilon$ decreases to zero. Now (iii) implies that $u_{1}=u$ in $\dot{K}_{3 r} \cap X_{p}$. We may take $U=\dot{K}_{2 r} \cap X_{p}$. The proof is complete.

COROLlary 4.1. Assume that the following condition is satisfied:

(H) $X$ is a nonempty bounded open subset of $\mathbf{R}^{2}$ and $F$ is a relatively closed subset of $X$ whose intersection with $X_{+}=\{(x, y) \in X: y \geq 0\}$ is a compact set $K$.

Then the following holds

(T) There exists an open neighborhood $U$ of $K$ such that every distribution $u \in D^{\prime}(U)$ satisfying

(1) $P(a,-a, c, k)=\left[\left(D_{x}+a x^{k} D_{y}\right)\left(D_{x}-a x^{k} D_{y}\right)-c x^{k-1} D_{y}\right] u=0$ in $X$,

(2) $\operatorname{supp} u \subset F$

also satisfies $u=0$ in $U$, when one of the following holds:

(i) $0 \neq a, c \in \mathbf{R} ; k a+c=0$,

(ii) $a, c \in \mathbf{R}, k$ odd and $k a+c<0$,

(iii) $0>a, c \in \mathbf{R}$; $k$ odd and for all $j=0,1,2, \ldots c \neq-2 a j(k+1)$, $c \neq-2 a[k+j(k+1)]$.

COROLlaRY 4.2. Assume that the following condition is satisfied:

(H) $X$ is a nonempty bounded open subset of $\mathbf{R}^{2}$ and $F$ is a relatively closed subset of $X$ whose intersection with

$$
X_{a, b, k}=\left\{(x, y) \in X: y \geq\left(\frac{a+b}{2}\right) \frac{x^{k+1}}{k+1}\right\}
$$

is a compact set $K$. 
Then the following holds:

(T) There exists an open neighborhood $U$ of $K$ such that every distribution $u \in D^{\prime}(U)$ satisfying

$$
\text { (1) } P(a, b, c, k) u=0 \quad \text { in } X ; \quad \text { (2) } \operatorname{supp} u \subset F
$$

also satisfies $u=0$ in $U$, when one of the following holds:

(i) $a, b, c \in \mathbf{R} ; a \neq b ; k((a-b) / 2)+c=0$

(ii) $a, b, c \in \mathbf{R} ; k$ odd and $k((a-b) / 2)+c<0$

(iii) $a, b, c \in \mathbf{R} ; a-b<0 ; k$ odd and for all $j=0,1,2, \ldots c \neq$ $j(b-a)(k+1), c \neq(b-a)[k+j(k+1)]$.

\section{REFERENCES}

[BP] B. Birkeland and J. Persson, The local Cauchy problem in $R^{2}$ at a point where two characteristic curves have a common tangent, J. Differential Equations, 30 (1978), 64-88.

[GT] A. Gilioli and F. Treves, An example in the solvability theory of linear PDE's, Amer. J. Math., 92 no. 2 (1974), 366-384.

[H] L. Hörmander, The Analysis of Linear Partial Differential Operators I: Distribution Theory and Fourier Analysis, Springer-Verlag (1983).

[N] S. Nakane, Propagation of singularities and uniqueness in the Cauchy problem at a class of doubly characteristic points, Comm. in PDE, 6(8) (1981), 917-927.

[K] L. Korbly, Uniqueness of solutions of partial differential equations when the initial surface is characteristic at a point, Proc. Amer. Math. Soc., 86 no. 4 (1982), 617-624.

[T1] F. Treves, Linear Partial Differential Equations With Constant Coefficients: Existence, Approximation and Regularity of Solutions, Math. and its Applications, 6 Gordon and Breach, New York (1966). , Discrete phenomena in uniqueness in the Cauchy problem, Proc. Amer. Math. Soc., 46 (1974), 229-233.

Received October 5, 1987 and in revised form February 26, 1988. The first author is on leave from: Universidade Federal de São Carlos (UFSCar), Departamento de Matemática, Caixa Postal 676, 13560-São Carlos-SP. Brazil.

Universidade de São Paulo

Caixa Postal 668

13560 São CARLos-SP.-BrazIL 


\section{PACIFIC JOURNAL OF MATHEMATICS \\ EDITORS}

\author{
V. S. VARADARAJAN \\ (Managing Editor) \\ University of California \\ Los Angeles, CA 90024 \\ HERBERT ClEMENS \\ University of Utah \\ Salt Lake City, UT 84112 \\ THOMAS ENRIGHT \\ University of California, San Diego \\ La Jolla, CA 92093
}

R. FINN

Stanford University

Stanford, CA 94305

HERMANN FLASCHKA

University of Arizona

Tucson, AZ 85721

VAUGhan F. R. JONES

University of California

Berkeley, CA 94720

STEVEN KERCKHOFF

Stanford University

Stanford, CA 94305

\section{ROBION KIRBY}

University of California

Berkeley, CA 94720

C. C. MOORE

University of California

Berkeley, CA 94720

HAROLD STARK

University of California, San Diego

La Jolla, CA 92093

\section{ASSOCIATE EDITORS}
R. ARENS
E. F. BECKENBACH
B. H. NEUMANN
F. WOLF
K. YOSHIDA

(1906-1982)

\section{SUPPORTING INSTITUTIONS}

UNIVERSITY OF ARIZONA

UNIVERSITY OF BRITISH COLUMBIA

CALIFORNIA INSTITUTE OF TECHNOLOGY

UNIVERSITY OF CALIFORNIA

MONTANA STATE UNIVERSITY

UNIVERSITY OF NEVADA, RENO

NEW MEXICO STATE UNIVERSITY

OREGON STATE UNIVERSITY

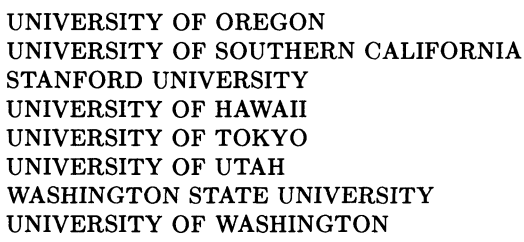

The Supporting Institutions listed above contribute to the cost of publication of this Journal, but they are not owners or publishers and have no responsibility for its content or policies.

\footnotetext{
Mathematical papers intended for publication in the Pacific Journal of Mathematics should be in typed form or offset-reproduced (not dittoed), double spaced with large margins. Please do not use built up fractions in the text of the manuscript. However, you may use them in the displayed equations. Underline Greek letters in red, German in green, and script in blue. The first paragraph must be capable of being used separately as a synopsis of the entire paper. In particular it should contain no bibliographic references. Please propose a heading for the odd numbered pages of less than 35 characters. Manuscripts, in triplicate, may be sent to any one of the editors. Please classify according to the scheme of Math. Reviews, Index to Vol. 39. Supply name and address of author to whom proofs should be sent. All other communications should be addressed to the managing editor, or Elaine Barth, University of California, Los Angeles, California 90024.

There are page-charges associated with articles appearing in the Pacific Journal of Mathematics. These charges are expected to be paid by the author's University, Government Agency or Company. If the author or authors do not have access to such Institutional support these charges are waived. Single authors will receive 50 free reprints; joint authors will receive a total of 100 free reprints. Additional copies may be obtained at cost in multiples of 50 .
}

The Pacific Journal of Mathematics is issued monthly as of January 1966. Regular subscription rate: $\$ 190.00$ a year (5 Vols., 10 issues). Special rate: $\$ 95.00$ a year to individual members of supporting institutions.

Subscriptions, orders for numbers issued in the last three calendar years, and changes of address should be sent to Pacific Journal of Mathematics, P.O. Box 969, Carmel Valley, CA 93924, U.S.A. Old back numbers obtainable from Kraus Periodicals Co., Route 100, Millwood, NY 10546.

The Pacific Journal of Mathematics at P.O. Box 969, Carmel Valley, CA 93924 (ISSN 0030-8730) publishes 5 volumes per year. Application to mail at Second-class postage rates is pending at Carmel Valley, California, and additional mailing offices. Postmaster: send address changes to Pacific Journal of Mathematics, P.O. Box 969, Carmel Valley, CA 93924.

PUBLISHED BY PACIFIC JOURNAL OF MATHEMATICS, A NON-PROFIT CORPORATION Copyright (C) 1989 by Pacific Journal of Mathematics 


\section{Pacific Journal of Mathematics}

\section{Vol. 136, No. 2 December, 1989}

A. K. Agarwal and David Bressoud, Lattice paths and multiple basic hypergeometric series ................................. 209

Adalberto Panobianco Bergamasco and Hermano de Souza Ribeiro, Uniqueness in a doubly characteristic Cauchy problem ........... 229

Thomas Curtis Craven and George Leslie Csordas, Jensen polynomials

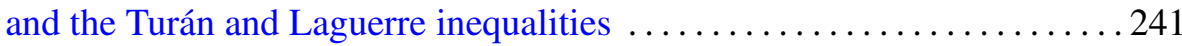

Gary R. Jensen and Marco Rigoli, Harmonic Gauss maps ............ 261

L. G. Kovács and Cheryl Elisabeth Praeger, Finite permutation groups with large abelian quotients $\ldots \ldots \ldots \ldots \ldots \ldots \ldots \ldots \ldots \ldots \ldots \ldots . \ldots \ldots$

Ken-ichi Maruyama, Localization of a certain subgroup of self-homotopy

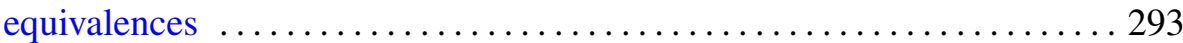

Tomasz Mazur, Canonical isometry on weighted Bergman spaces ......... 303

Bernt Karsten Oksendal, A stochastic Fatou theorem for quasiregular

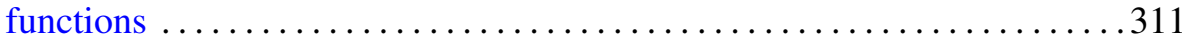

Ian Fraser Putnam, The $C^{*}$-algebras associated with minimal homeomorphisms of the Cantor set ........................ 329

Tom Joseph Taylor, Some aspects of differential geometry associated with

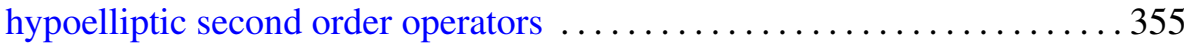

Tom Joseph Taylor, Off diagonal asymptotics of hypoelliptic diffusion equations and singular Riemannian geometry 